\title{
Lacrimal Gland Carcinoma pT4b TNM Finding v8
}

National Cancer Institute

\section{Source}

National Cancer Institute. Lacrimal Gland Carcinoma pT 4b TNM Finding v8. NCI

Thesaurus. Code C140799.

Lacrimal gland carcinoma with involvement of adjacent structures, including sinuses,

temporal fossa, pterygoid fossa, superior orbital fissure, cavernous sinus, or brain and

measuring more than $2 \mathrm{~cm}$ but $4 \mathrm{~cm}$ or less in greatest dimension. (from AJCC 8th Ed.) 